\title{
AS ABORDAGENS MARKET-DRIVEN E MARKET-DRIVING DE ORIENTAÇÃO PARA O MERCADO E INOVAÇÃO: PROPOSIÇÃO DE UM MODELO INTEGRADO
}

\author{
Valter Afonso Vieira \\ valterafonsovieira@yahoo.com \\ Universidade Federal do Paraná - PR/Brasil \\ Claudio Zancan \\ czancan@unb.br \\ Universidade de Brasília - Brasília/DF
}

Recebido em 13/10/2009

Aprovado em 30/11/2010

Disponibilizado em 01/08/2011

Avaliado pelo sistema double blind review

Revista Eletrônica de Administração

Editor: Luís Felipe Nascimento

ISSN 1413-2311 (versão on-line)

Editada pela Escola de Administração da Universidade Federal do Rio Grande do Sul.

Periodicidade: Quadrimestral

Sistema requerido: Adobe Acrobat Reader.

\section{INTRODUÇÃO}

Em quase duas décadas de intensa pesquisa, o tema de Orientação para o Mercado consubstanciou-se em um campo teórico de inegável desenvolvimento. Nesse contexto, observações sobre o relacionamento entre uma postura de 'orientação e um conseqüente desempenho' superior firmaram-se como o objetivo precípuo das investigações na área. Durante o desenvolver desse relacionamento, direcionamentos recentes da teoria de orientação para o mercado têm apontado para a natureza eminentemente reativa das estratégias relacionadas. Argumenta-se que a abordagem tradicional - market-driven - seja excessivamente determinista e de forte conotação adaptativa perante o ambiente externo. Assim, essa visão passa a ser complementada por uma nova perspectiva - market-driving pressupondo a possibilidade de que as estruturas e o comportamento do mercado possam ser modelados pelas organizações.

Entretanto, ainda são incipientes os esforços na construção de um modelo de referência que possa nortear futuras pesquisas no âmbito dessas duas estratégias em conjunto. É nessa problemática que se insere o objetivo do presente trabalho, em vistas de apresentar 
um modelo teórico integrado para a estratégia de orientação para o mercado, justamente contemplando as duas abordagens aludidas. Sobre o modelo lançam-se as proposições de pesquisa pertinentes.

O encaminhamento do artigo dar-se-á da seguinte forma: primeiramente, apresentamse considerações referenciais sobre o modelo clássico de orientação para o mercado (marketdriven). Descrevem-se na seqüência as críticas que se fazem a esse modelo, mais especificamente relativas à sua natureza customer-led. Somando-se a essas descrições, a abordagem market-driven é descrita. Os tópicos relacionados servem de substrato para a construção das proposições de pesquisa e respectivo modelo integrado proposto. Finalmente, exibem-se as conclusões advindas do presente trabalho, as considerações que justificam a propriedade acadêmica e gerencial da questão pesquisada, bem como se descreve uma possível agenda para investigações futuras.

\section{Revisão Teórica}

\subsection{A Abordagem Market-Driven}

A abordagem market-driven ${ }^{1}$ representa os construtos e princípios tradicionais de orientação para o mercado. Teoria de importância inconteste no panorama do marketing nos últimos quinze anos, a orientação para o mercado constrói sólido edifício conceitual a partir da década de noventa. Nesse contexto, tem sua relação com a performance investigada de forma exaustiva em inúmeros estudos nos mais diversos ambientes e condições, os quais apontam quase que invariavelmente para uma correlação positiva entre os dois construtos (NARVER; SLATER, 1990; PITT, CARUANA; BERTHON, 1996; AVLONITIS; GOUNARIS, 1997; BHUIAN, 1998; SUBRAMANIAN; GOPALAKRISHNA, 2001; MENNA, 2001; GAVA, 2007; GAVA; SILVEIRA, 2007). Mais recentemente, estudos descritivos (LANGERAK, 2002) e meta-analíticos (CANO; CARRILLAT; JARAMILLO, 2004; KIRCA, JAYACHANDRAN; BEARDEN, 2005), não obstante corroborem a correlação aludida, citam inúmeras pesquisas em que a mesma não apresentou significância, bem como a intensa variabilidade dos resultados. A questão, pois, continua em aberto.

A orientação para o mercado transcende a clássica orientação para marketing (marketing orientation), ao propor que outros públicos - concorrência, fornecedores, governo

\footnotetext{
${ }^{1}$ Desde que a literatura passou a questionar a natureza reativa da orientação para o mercado, a consagrada denominação market orientation (KOHLI E JAWORSKI, 1990; NARVER e SLATER, 1990) tem sido substituída pela expressão market-driven, com a clara intenção de contrastá-la com o termo market-driving. Assim, market-driven e market-oriented são nomenclaturas que dizem respeito ao mesmo fenômeno. Note-se que o termo market-driven não é propriamente uma novidade no campo, considerado sinônimo de market orientation desde Shapiro (1988), bem como em Ruekert (1992), Day (1994) e Webster (1994).
} 
As abordagens market-driven e market-driving de orientação para o mercado e inovação: proposição de um modelo integrado

- também sejam contemplados na análise externa. Essa concepção encontra-se no cerne da literatura seminal de orientação (KOHLI; JAWORSKI, 1990, p.3); o foco no cliente constituise em um dos pilares - mas não o único - do construto (NARVER; SLATER, 1990). É Shapiro (1988) quem inicialmente caracteriza as organizações market-oriented, baseadas nas informações de mercado, na tomada conjunta de decisões e na coordenação interfuncional. Essa visão é consolidada por Kohli e Jaworski (1990) na clássica estrutura "geração de inteligência, disseminação e capacidade de resposta" e também por Narver e Slater (1990), que apesar de conceberem a orientação para o mercado sob a ótica cultural, também alocam as informações e sua importância ao âmago do construto. A ênfase na informação de mercado continua presente na definição de Day (1994), que alça as capacidades de senso de mercado e ligação com os clientes como cruciais para as organizações que se pretendem market-driven.

Importante perceber, assim, que a orientação para o mercado nasce e se dissemina fortemente como uma perspectiva baseada na informação (HARRIS; CAI, 2003), direcionando essa coleta aos clientes e respondendo ao mercado com base nas informações geradas (LAFFERTY; HULT, 2001). Amplia-se o foco sobre o cliente, preocupando-se também com outros atores do cenário competitivo, a abordagem de orientação mantém os consumidores e seus anseios no cerne das atenções estratégicas. E é justamente sobre esse foco que recaem as críticas ao caráter reativo do construto, contexto no qual desponta a abordagem market-driving.

Como ressaltam Jaworski, Kohli e Sahay (2000), o histórico de pesquisas sobre orientação para o mercado denota uma interpretação desbalanceada de sua natureza. Em específico, observam que o construto acabou sendo tomado como sinônimo de uma simples orientação para o cliente, embora não tenha sido essa a intenção que o originou. A proximidade com o cliente (staying close to the customer), a adaptação às mudanças em seu comportamento e a adequação da oferta às suas necessidades, são os paradigmas da postura market-driven. A crítica market-driving vem justamente enfatizar que ser market-driven não é o único caminho existente para orientar-se ao mercado.

Assim, a literatura de orientação para o mercado passa a englobar também essa abordagem emergente, lançando o debate market-driving como uma alternativa complementar. Se recente em sua concepção, tem suas raízes conceituais em trabalhos desenvolvidos no âmbito da chamada gestão do ambiente (environmental management), representada por estudos como os de Zeithaml e Zeithaml (1984) e Varadarajan, Clarck e Pride (1992). Ainda, trabalhos como os de Hamel e Prahalad (1991), Christensen e Bower 
(1996) e Slater e Narver (1998), ampliam o substrato sobre o qual a abordagem marketdriving alicerça seus argumentos.

Importante ressaltar que a crítica market-driving traz consigo considerações a respeito da chamada postura customer-led. Em outras palavras: a reatividade contida na postura market-driven é vista como reprodutora de padrões estratégicos demasiadamente voltados aos clientes da organização.

Os tópicos até aqui relatados são fundamentais para que se compreenda a crítica aposta pela abordagem market-driving. Em conseqüência, é sobre eles que se encaminham as considerações que seguem.

\subsection{A Abordagem customer-led}

O foco no cliente - e mais genericamente, no mercado - e suas necessidades expressas, é quem alimenta grande parte da crítica à natureza reativa do construto de orientação. Nesse contexto, a orientação estratégica market-driven é vista como reprodutora do posicionamento customer-led, sendo por isso posta em cheque enquanto estratégia de mercado. Os efeitos limitadores de tal postura, essencialmente reativa e voltada à adaptação, refletem boa parte dos argumentos utilizados pela literatura market-driving.

Ser customer-led é ser "guiado pelo cliente", monitorando e respondendo às suas necessidades expressas (SLATER; NARVER, 1998). Já Hamel e Prahalad (1991) apontavam para os riscos dessa atitude, naquilo que denominaram de "tirania do mercado atendido" (tyranny of the served market). Enquanto algumas empresas se esforçam para serem orientadas ao mercado - adaptando-se às condições aí existentes - concorrentes de classe mundial buscam criar novos mercados a partir do uso avançado de tecnologias. Essas organizações detêm a habilidade de imaginar (imaginatives companys) realidades não existentes, criando novos espaços competitivos para além do horizonte de rivalidade convencional. Faz isso a partir de uma visão ampla de negócio, baseada em competências e não no portfólio de produtos, perseguindo inovações radicais e revolucionárias e preocupando-se mais em gerar necessidades do que simplesmente tentar detectá-las. Ainda, são organizações abertas à experimentação e ao erro, aprendendo evolutivamente (FIGUEIREDO, 2003) à medida que avançam nesse processo de criação (expeditionary marketing).

Também Christensen e Bower (1996) denotam os malefícios de uma postura customer-led. Observam que "ouvir os clientes em demasia" é um limitador estratégico marcante, o qual diminui as possibilidades de posicionamento por parte das firmas. Seu 
As abordagens market-driven e market-driving de orientação para o mercado e inovação: proposição de um modelo integrado

corolário é o aumento das probabilidades de fracasso estratégico, principalmente em períodos de mudanças no comportamento do mercado. A partir de pesquisa na indústria de computadores, os autores observam que a maior parte dos recursos organizacionais são empregados em uma perspectiva market-driven, voltada à inovação incremental e ao exclusivo atendimento das demandas expressas e atuais do mercado. Em consequiência, negligenciam-se possibilidades inovativas radicais (NELSON; WINTER, 2005), dispostas a modificar os padrões de mercado vigentes.

Naquilo que determinou por "dilema da inovação", Christensen (2001) defende a tese de que o desenvolvimento incremental de tecnologias - melhorando os produtos nas formas pretendidas pelos clientes - é um dos elementos do fracasso de muitas organizações. Extensa pesquisa do autor na indústria de hard-disks é o substrato dos argumentos levantados. Christensen (2001) ilustra por que líderes na fabricação de drives acabaram por tornarem-se ineficientes no lançamento de inovações de ruptura, embora fossem tecnologicamente capazes de produzi-las. Para o autor, justamente o foco em demasia nas necessidades atuais dos clientes acabou por tornar essas empresas "cativas de seus consumidores", e por isso, incapazes de vislumbrar mercados ainda não existentes.

\subsection{A Abordagem Market-Driving}

Buscando referenciais nos estudos até aqui descritos, a abordagem market-driving emerge no panorama da orientação para o mercado no final dos anos noventa. Incorporando a crítica à orientação customer-led, inserem ao debate novos elementos, na tentativa de consolidar uma visão estruturada do tema. Assim, a abordagem market-driving intitula-se desde o princípio como uma estratégia orientada ao mercado de natureza pró-ativa, advogando a modificação das condições ambientais existentes. Passa a criticar a natureza acentuadamente determinista imputada à orientação market-driven, tendo-a como demasiadamente adaptativa (KUMAR, SCHEER, KOTLER, 2000). De forma mais específica, a abordagem market-driving aventa a possibilidade de que as estruturas e/ou os comportamentos do mercado possam ser modelados pelas organizações (JAWORSKI; KOHLI; SAHAY, 2000).

Instaura-se assim o contraste entre duas abordagens distintas no panorama da orientação para o mercado: a orientação market-driven representa a tomada de decisões a partir do reconhecimento das preferências e comportamentos de clientes, consumidores e concorrentes; é uma filosofia de reação, baseada em um contexto "dado" e aceito como tal. Baseia-se no aprendizado simplesmente adaptativo e limita-se à inovação incremental REAd - Edição 69 - Vol. 17 - N 2 - maio/agosto 2011 - p. 480-501 
(FIGUEIDO, 2003; TARNOVSKAYA; ELG; BURT, 2005). Ser market-driving, por outro lado, implica uma postura mais agressiva e baseada na pró-atividade, com o nítido objetivo de influenciar as estruturas do mercado e/ou o comportamento de seus players. Relaciona-se à inovação radical, à tomada de risco e à antecipação às dinâmicas de mercado.

É Kumar (1997) quem primeiro menciona o termo market-driving em estudo sobre mudanças radicais no setor de varejo. Observando as profundas transformações nessa indústria nos anos noventa, o autor observa a proeminência de empresas como Amazon, Body Shop, Wal-Mart e IKEA. Destaca a pró-atividade das estratégias por elas adotadas, no intuito de modificar as condições de mercado existentes, bem como dirigir (driving) os comportamentos e hábitos dos consumidores. Conclui, apontando para a obsolescência de estratégias exclusivamente reativas ao mercado (market-driven), pautadas pela adaptação às necessidades atuais dos clientes.

À mesma época, Carpenter, Glazer e Nakamoto (1997) oferecem construção ao tema, e o fazem a partir da perspectiva do comportamento do consumidor. Se as preferências dos consumidores e seu processo de decisão - como denotam - são também dependentes do contexto, então a recriação das condições aí vigentes torna-se tarefa fundamental para a estratégia de marketing. Nessa perspectiva, modelar o contexto em que os clientes se inserem significa modelar suas próprias preferências e comportamentos. Na dimensão market-driven o cliente se move primeiro, estabelecendo estruturas em relação às quais as organizações reagem; naquela market-driving o movimento parte das firmas, que oferecem estratégias as quais os clientes irão responder.

O trabalho já citado de Kumar (1997) amplia-se em parceria com outros autores (KUMAR; SCHEER; KOTLER, 2000), passando a dimensionar os fatores intraorganizacionais que facilitam a implementação de uma estratégia market-driving. Paralelamente no tempo, Jaworski, Kohli e Sahay (2000) preocupam-se em analisar de que forma as condições de mercado podem ser modeladas pelas organizações. Deve ser notada a motivação distinta dos dois trabalhos: enquanto Kumar, Scheer e Kotler (2000) absorvem-se em identificar os aspectos organizacionais necessários à articulação market-driving portanto, em uma perspectiva interna - Jaworski, Kohli e Sahay (2000) analisam de que forma as organizações podem modificar as condições de mercado vigentes, voltando suas considerações mais para as ações estratégicas do que para os antecedentes relacionados a sua formulação.

Contextualiza-se, assim, o campo teórico da estratégia market-driving a partir das perspectivas internas e externas relatadas (HARRIS; CAI, 2003; TARNOVSKAYA; ELG; REAd - Edição 69 - Vol. 17 - N 2 - maio/agosto 2011 - p. 480-501 
As abordagens market-driven e market-driving de orientação para o mercado e inovação: proposição de um modelo integrado

BURT, 2005). Em outras palavras, é sobre as considerações de Jaworski, Kohli e Sahay (2000) e Kumar, Scheer e Kotler (2000) que os estudos posteriores dão curso ao debate e baseiam suas proposições. Esses estudos, embora representem avanços iniciais para a teoria em curso, ainda estão longe de fornecer um modelo estratégico integrado para a postura de orientação para o mercado. Portanto, guardando essa realidade, apresentam-se proposições de pesquisa idealizadas para o modelo em tese, baseadas na intensiva revisão de literatura até aqui descrita.

\section{Proposições de Pesquisa e Modelo Teórico}

Inicia-se a construção das proposições a partir da abordagem customer-led. Se a crítica construída pela abordagem market-driving repousa em grande parte nesta questão, justifica-se aqui sua propriedade ao tema. Nesse contexto, retomam-se as análises descritas de Christensen e Bower (1996) e Hamel e Prahalad (1991), ligando a ênfase excessiva no cliente de forma inequívoca a uma postura de mercado reativa.

Em sua origem teórica (Kohli e Jaworski, 1990), o construto de orientação para o mercado tem o foco no cliente como "mais um elemento" no contexto da estratégia voltada ao ambiente de negócios. Assim, enquanto não afasta a importância do cliente no contexto da estratégia, a abordagem de orientação amplia a visão de mercado, chamando a atenção para outros atores do cenário competitivo. Pode-se afirmar, dessa forma, que o foco no cliente seja variável necessária - mas não suficiente - para a caracterização de uma estratégia de orientação para o mercado.

Com base nisso, concebe-se aqui um relacionamento $\cap$ shaped-form entre as posturas market-driven e customer-led. Em outras palavras, entende-se que quanto mais orientada para o mercado a organização é (na forma market-driven), mais ela será guiada pelo cliente (customer-led). Todavia, defende-se aqui a tese de que essa relação possua um ponto crítico, quando a atenção no cliente passa a representar uma desatenção noutros componentes do construto de orientação.

Desta feita, presume-se que em um determinado momento de estar "ouvindo os clientes em demasia”, a organização começa a ficar menos orientada pelo mercado, uma vez que passará a negligenciar outros atores do cenário de negócios. Na verdade, se Christensen e Bower (1996) e Hamel e Prahalad (1991) estiverem corretos, existe uma relação curvilinear entre as abordagens market-driven e customer-led, representada na figura 1. Portanto a primeira proposição de pesquisa é: 
$P_{1}$ : A orientação market-driven possui uma associação em $\cap$ shaped-form com a postura customer-led.

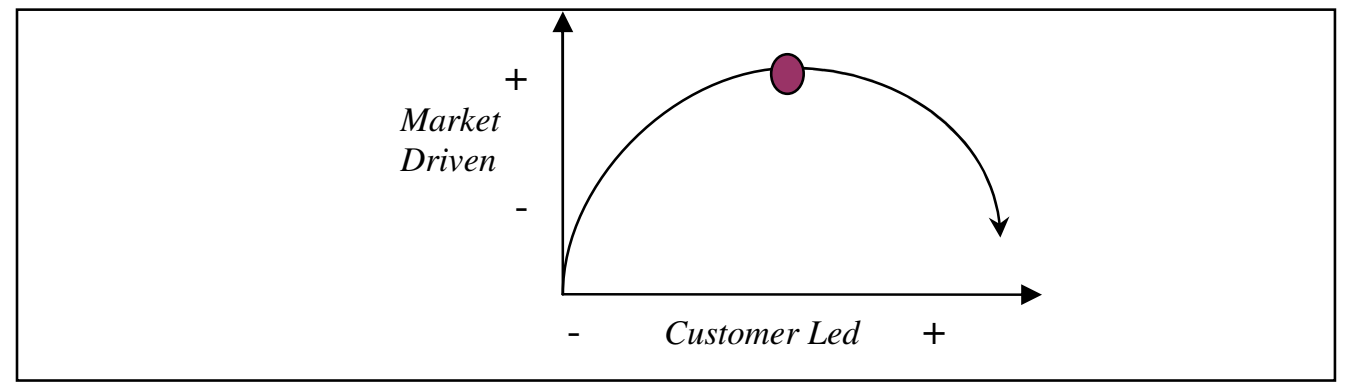

Figura 1: Exemplificação da associação em $\cap$ shaped-form

Fonte: os autores

Não obstante abranger a postura customer-led em seu âmago, a estratégia de orientação para o mercado dela se diferencia. Na acepção de Slater e Narver (1998; 1999), as duas abordagens não se confundem - ou pelo menos, não deveriam se confundir. Na verdade, ambas as abordagens representam diferentes maneiras de se entender o mercado, duas lógicas distintas no panorama da orientação. Isso, pois, a primeira concerne ao atendimento dos desejos expressos dos clientes, consistindo em uma perspectiva de curto prazo e de natureza eminentemente reativa. A segunda - market-oriented - busca identificar também as necessidades latentes dos clientes e satisfazê-las, focando no longo prazo e premiando a próatividade. Nesse sentido, Slater e Narver $(1998$; 1999) salientam, e isso é importante, que os dois posicionamentos estratégicos não são excludentes, mas complementares, e de eficácia contingente ao contexto de aplicação.

Por conseqüência, se a tese de Slater e Narver $(1998 ; 1999)$ estiver correta e se ambas as estratégias representam diferentes maneiras de se entender o mercado, poder-se-ia esperar uma correlação baixa entre ambas ou mesmo uma discriminação, dado que uma é em longo prazo e outra é em curto prazo. Assim sendo, a próxima proposição de pesquisa pressupõe que:

$P_{2}$ : A orientação market-driven é um construto distinto daquele customer-led.

$\mathrm{O}$ aspecto da complementaridade aparece também na relação das posturas marketdriving e market-driven. Esse caráter complementar é evidenciado por Jaworski, Kohli e Sahay (2000) e corroborado empiricamente no estudo de caso de Harris e Cai (2002).

Se Harris e Cai (2002) constatam o caráter complementar das abordagens marketdriving e market-driven, dão a ele entendimento adicional. Nesse particular, sustentam a natureza seqüencial das duas estratégias, no sentido de que as duas completam-se, mas em 
As abordagens market-driven e market-driving de orientação para o mercado e inovação: proposição de um modelo integrado

momentos temporais distintos: o sucesso de uma estratégia market-driving requer, a posteriori, a aplicação de ações market-driven.

Note-se que essa concepção coaduna com o raciocínio de Kumar, Scheer e Kotler (2000), para quem as organizações podem ser market-driving num primeiro estágio de desenvolvimento de mercado, ajustando posteriormente esse posicionamento para uma estratégia market-driven. Em suma, se as duas completam-se, mas em momentos temporais distintos, é esperada uma condição de construto preditor da abordagem market-driving. É com esse entendimento que se projeta a próxima proposição:

$P_{3}$ : A orientação market-driving tem impacto positivo sobre a estratégia marketdriven, uma vez que é antecessora dessa.

O contraponto market-driven / market-driving tem em Day (1999) sua crítica mais contundente. $\mathrm{O}$ autor entende que uma estratégia de orientação para o mercado legítima e eficaz traga implicitamente uma conotação pró-ativa em seu âmago. Assim, não há o que se falar em duas abordagens paralelas de orientação para o mercado; uma empresa market-driven será, em decorrência e naturalmente, também market-driving.

Note-se que o discurso de Day (1999) não afasta a reatividade em pauta; apenas a tem como uma das dimensões do construto de orientação para o mercado. Observa que a crítica market-driving equivoca-se ao negligenciar a possibilidade de que a busca de informações sobre os clientes possa também se debruçar sobre aquelas não explicitadas, por meio de investigações mais profundas a respeito das opiniões e comportamentos latentes dos consumidores. O mesmo equívoco que, no âmbito da teoria de orientação para o mercado, afasta a possibilidade de que essa possa aglutinar as perspectivas reativas e pró-ativas em sua formulação e implementação.

Assim, para Day (1999), orientar-se "ao" mercado e orientar "o" mercado não são posições excludentes: organizações podem - e devem - liderar e seguir seus clientes ao mesmo tempo, orientando-se concomitantemente pelo mercado (market-pull) e pela inovação (technology-push).

Primeiramente, entendesse aqui que o argumento de Day (1999), apesar de estruturarse sobre premissas válidas, não seja satisfatório para o contexto. Em primeiro lugar, se é concebível que uma postura market-driven seja, implicitamente, também market-driving, tal possibilidade não é uma prova indefectível de sua ocorrência. A adoção de uma postura market-driven não pressupõe, necessariamente, que esteja permeada por ações voltadas a modelar as estruturas e o comportamento do mercado. 
Em segundo lugar, chama-se atenção que as próprias escalas de orientação para o mercado tradicionais (MARKOR; MKTOR) atestam o desequilíbrio em prol da postura adaptativa. Terceiro e último ponto, a análise da literatura market-driving em nenhum momento apregoa o caráter excludente dessa abordagem em relação àquela market-driven. Pelo contrário, o equilíbrio entre posturas reativas e pró-ativas no âmbito da orientação para o mercado - "estratégia dual" - é visto como o estado da arte da estratégia competitiva (JAWORSKI, KOHLI e SAHAY, 2000; NARVER, SLATER e MACLACHLAN, 2004). Nessa circunstância, a próxima suposição lançada é:

$P_{4}:$ A orientação market-driving tem impacto positivo sobre uma estratégia marketdriven, uma vez que ambas guardam uma relação de complementaridade.

O relacionamento da orientação para o mercado com a performance empresarial é um aspecto que nasce com a própria teoria. Kohli e Jaworski (1990) já discutiam o desempenho organizacional superior como consequiência da orientação para o mercado, contemporaneamente aos desenvolvimentos iniciais do construto.

Nesse contexto, a estratégia market-driven tem sua relação com a performance investigada de forma exaustiva em inúmeros estudos nos mais diversos ambientes e condições, os quais apontam quase que invariavelmente para uma correlação positiva entre os dois construtos (NARVER; SLATER, 1990; PITT; CARUANA; BERTHON, 1996; AVLONITIS; GOUNARIS, 1997; BHUIAN, 1998; SUBRAMANIAN; GOPALAKRISHNA, 2001; MENNA, 2001).

Assim, verificar empiricamente a influência da orientação para o mercado marketdriven sobre a performance constituiu-se em aspecto significativo das investigações na área, em uma agenda de pesquisa extremamente prolífica e palco de incandescente debate. De forma geral, a primeira sucessão de estudos delineou uma relação positiva entre a postura de orientação para o mercado e o conseqüente desempenho superior das organizações (NARVER; SLATER, 1990; SLATER; NARVER, 1994; PELHAM， 1997). O relacionamento da orientação para o mercado com a performance empresarial é um aspecto que nasce com a própria teoria. Assim, a literatura indica uma associação descrita na seguinte forma:

$P_{5}:$ A estratégia market-driven de orientação para o mercado relaciona-se positivamente com a performance de mercado.

Se a relação da abordagem de orientação para o mercado com a performance é um dos pilares da pesquisa no campo desde sua origem (LANGERAK, 2002; KIRCA, JAYACHANDRAN; BEARDEN, 2005), no âmbito específico da estratégia market-driving REAd - Edição 69 - Vol. 17 - N 2 - maio/agosto 2011 - p. 480-501 
As abordagens market-driven e market-driving de orientação para o mercado e inovação: proposição de um modelo integrado

isso não é diferente. Assim, os estudos postulam a relação direta entre as ações voltadas a modelar o mercado e a construção de vantagem competitiva sustentável (JAWORSKI; KOLHI; SAHAY, 2000; KUMAR; SCHEER; KOTLER, 2000; HARRIS; CAI, 2002).

Jaworski, Kohli e Sahay (2000) dispõem que a aplicação de uma estratégia marketdriving esteja relacionada de forma marcante a performances superiores, constituindo-se em um desafio para a administração. Hills e Sarin (2003) descrevem os benefícios de uma postura pró-ativa perante o ambiente no setor de alta tecnologia, representando um novo paradigma para a inovação de produtos. O esquema conceitual de Carrillat, Jaramillo e Locander (2004) intensifica esse entendimento, arrolando a performance superior como elemento consequente de uma estratégia market-driving.

A despeito dessas constatações e, diante do pequeno número de estudos empíricos desenvolvidos no âmbito da abordagem market-driving, entende-se que existam limitações sobre a realidade dessa suposta relação. Entendendo-se a importância de averiguar esse fato, tem-se a proposição que segue:

$P_{6}:$ A estratégia market-driving de orientação para o mercado relaciona-se positivamente com a performance de mercado.

A complementaridade já aludida entre as duas abordagens de orientação também tangencia o fator desempenho. Jaworski, Kohli e Sahay (2000) salientam que estratégias market-driven e market-driving, habilmente conciliadas, geram perspectivas de maior sucesso competitivo para as organizações. Posição já assumida por Day (1999), para quem uma efetiva orientação para o mercado supõe o equilíbrio entre ações reativas e pró-ativas em relação ao ambiente de negócios. Saliente-se que essa capacidade de gerenciar simultaneamente as necessidades do hoje e as oportunidades do amanhã tem sido alçada como o estado da arte da estratégia de mercado (HAMEL; PRAHALAD, 1991).

Postula-se que as organizações podem, e devem, simultaneamente, impetrar esforços no sentido de atender o mercado de forma reativa, ao mesmo tempo e que procuram modificar as regras e comportamentos ali presentes (CONNOR, 1999). Para Johnson, Lee, Saini e Grohmann (2003), uma empresa pode ser market-driven no curto prazo e market-driving no longo prazo ou vice-versa. Entendimento atestado empiricamente por Harris e Cai (2003), denotando que as duas estratégias suportam-se mutuamente e são assim reciprocamente indispensáveis, além de seqüenciais: a manutenção do sucesso de uma estratégia marketdriving requer, a posteriori, a aplicação de ações market-driven.

Coaduna com essa concepção o raciocínio de Kumar, Scheer e Kotler (2000), postulando que as organizações podem ser market-driving em um primeiro estágio de REAd - Edição 69 - Vol. 17 - N 2 - maio/agosto 2011 - p. 480-501 
desenvolvimento de mercado, ajustando posteriormente esse posicionamento para uma estratégia market-driven.

Entende-se, portanto, que o desempenho de mercado resultante de uma estratégia market-driving, seja moderado pela aplicação conjunta ou seqüencial de uma estratégia market-driven. A literatura, entretanto, ainda não consolidou a compreensão sobre quais das duas relações apresente uma influência mais significativa a esse respeito, se a primeira ou a segunda. Assim, a existência desses dois entendimentos no âmbito da relação entre as duas abordagens levou à articulação das seguintes proposições:

$P 7_{a}$ : O desempenho de mercado oriundo de uma estratégia market-driving é moderado pela aplicação conjunta de uma estratégia market-driven.

$P 7_{b}$ : O desempenho de mercado oriundo de uma estratégia market-driving é moderado pela aplicação seqüencial de uma estratégia market-driven.

Por fim, pesquisas indicam que a orientação para o mercado impacta em melhores resultados da organização, tanto de mercado quanto de aspectos financeiros (DESHPANDÉ; FARLEY; WEBSTER, 1993; WRIGHT, PEARCE; BUSBIN, 1997; HOMBURG; PFLESSER, 2000). Especificamente, a performance é definida como a eficácia das atividades de marketing da organização e é medida por meio da imagem no mercado (positiva ou negativa), nível de satisfação e lealdade dos clientes (HOMBURG; PFLESSER, 2000). Assim, o desempenho de mercado influenciaria diretamente o desempenho financeiro, medido por meio do retorno sobre as vendas e lucratividade da organização (RÉVILLION, 2003). Portanto:

$P_{8}:$ O desempenho de mercado tem um efeito positivo no desempenho financeiro da empresa.

Para Hooley et al. (2005), o acréscimo da orientação para o mercado induz ao desenvolvimento das capacidades de conexão ou relacionamentos com consumidores (DAY, 1994) e ao avanço da habilidade no desenvolvimento e lançamento de novos produtos e serviços, entendido como uma capacidade de inovação de mercado (SLATER; NARVER, 1995; HAN; KIM; SRIVASTAVA, 1998). Por outro ponto, a capacidade de inovação (DOSI, 2006), por sua vez, pode ser considerada como um antecedente do sucesso de novos produtos (HURLEY; HULT, 1998). Indo além, a habilidade no desenvolvimento de novos ou melhores produtos e serviços, através do sucesso de novos produtos (performance da inovação) (HAN, KIM; SRIVASTAVA, 1998), culmina em níveis mais efetivos de satisfação e lealdade de clientes (MAVONDO; CHIMHANZI; STEWART, 2005), em melhores resultados de volume 
As abordagens market-driven e market-driving de orientação para o mercado e inovação: proposição de um modelo integrado

de vendas e participação de mercado (HOOLEY et al., 2005), bem como em um impacto positivo na performance financeira (HURLEY; HULT, 1998) . Por decorrência:

$P_{9}:$ O nível de orientação para o mercado impacta positivamente na capacidade de inovação de mercado.

$P_{10}$ : A capacidade de inovação de mercado tem efeito positivo e direto sobre a performance de inovação.

$P_{11}$ : A performance de inovação afeta positivamente a performance de mercado.

$P_{12}$ : A performance de inovação afeta positivamente a performance financeira.

Fazendo uma análise da literatura, este artigo sugere uma relação de mediação da variável processo de inovação (HAN; KIM; SRIVASTAVA, 1998; HURLEY; HULT, 1998). A definição de mediação $(M e d)$ possui mais de 50 anos na literatura e não é recente. A sua conceitualização foi avançada por Rozeboom (1956) para construtos que tem relação linear. Assim, a definição é: $M e d$ é uma mediadora da relação probabilística $Y=f(X)$ se Med é uma função probabilística de $X$ (i.e., $M e d=f[X])$ e $Y$ é uma função probabilística de $M e d$ (i.e., $\hat{Y}$ $=f[\mathrm{Med}])$, onde $X, Y$ e $\mathrm{Med}$ representam construtos diferentes.

Kirca, Jaachandran Bearden (2005) encontraram uma relação de mediação da variável processo de inovação, contudo, o estudo desses pesquisares é limitado por ser de uma "base" de meta-análise e não de um estudo de campo efetivo. Assim, futuros trabalhos devem verificar o argumento do papel "bloqueador" do processo de inovação na relação entre orientação para o mercado e a performance organizacional. Acredita-se que orientação para o mercado é preditor significativo da performance organizacional em uma relação linear direta, entretanto, quando a variável processo de inovação é acrescida na equação, a associação torna-se não significativa, devido forte papel da inovação. Portanto:

$P_{13}$ : A relação entre orientação para o mercado e a performance organizacional é mediada pelo processo de inovação.

Posteriormente à construção exaustiva do arcabouço teórico, com base nos estudos até aqui realizados no âmbito da estratégia market-driving e market-driven, e levando em consideração as proposições de pesquisa advogadas, chega-se ao modelo explicativo proposto (Figura 2). O mesmo está assim definido: 


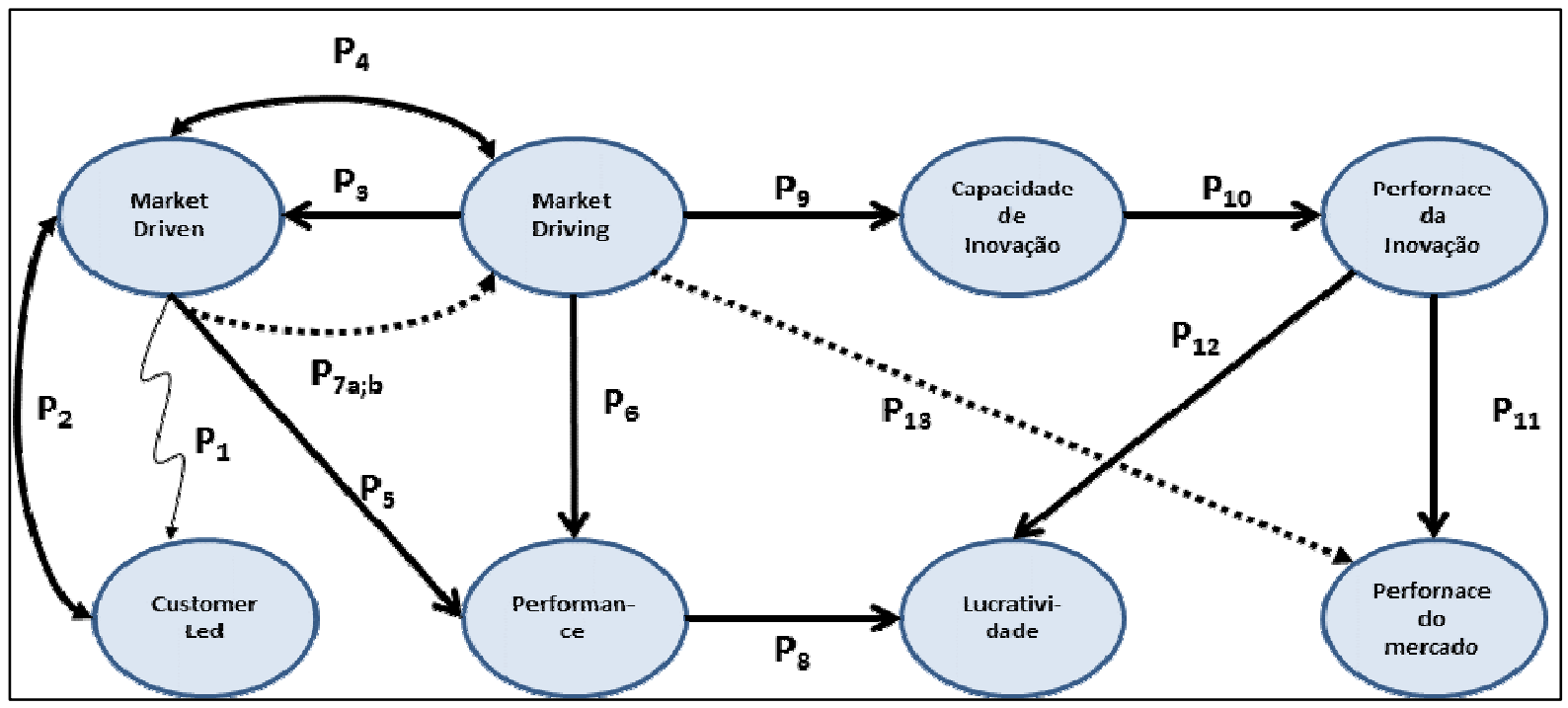

Figura 2: Modelo Integrado Proposto para a Estratégia de Orientação ao Mercado Fonte: os autores

O modelo teórico apresentado, com seus antecedentes, efeitos curvilineares $\left(\mathrm{P}_{1}\right)$ e conseqüências, produto final do presente trabalho, denota os elementos necessários para a formulação de uma estratégia de orientação para o mercado permeada pelas posturas marketdriven e market-driving de orientação para o mercado. $\mathrm{O}$ objetivo é verificar empiricamente no futuro como as estratégias se comportam entre si. Algumas relações são claras e já solidificadas pela teoria $\left(\mathrm{P}_{8}\right)$; outras necessitam de uma compreensão melhor para amadurecerem $\left(\mathrm{P}_{1}, \mathrm{P}_{2}, \mathrm{P}_{7 \mathrm{a} ; \mathrm{b}}\right)$. No que tange a relação entre orientação para mercado e inovação tem ganhado corpo nos últimos anos. Este ensaio teórico propõe que orientação para o mercado tem impacto na capacidade de inovação $\left(\mathrm{P}_{9}\right)$, uma vez que compreender o cliente, e suas exigências, é indicador para elaborar produtos novos. Essa capacidade de inovação gera por consequência performance de inovação $\left(\mathrm{P}_{10}\right)$, pois a capacidade introduz, por vezes, capacidade de aprendizagem ${ }^{2}$ das organizações (FIGUEIREDO, 2003). Essa capacidade de inovação tende a gerar melhor performance e $\left(\mathrm{P}_{11}\right)$, paralelamente, lucros $\left(\mathrm{P}_{12}\right)$. Portanto, a proposta principal do estudo é sugerir o modelo integrado da estratégia de orientação para o mercado para que novas pesquisas o refinem cada vez mais.

\footnotetext{
${ }^{2}$ Para Ipiranga (2006), em relação à análise das práticas e implicações do processo de aprendizagem e considerando as contribuições seminais sobre as atividades de "aprendendo fazendo" (learning by doing) (ARROW, 1962), "aprendendo usando" (learning by using) e "aprendendo a aprender" (learning to learn) uma boa quantidade de pesquisas foi desenvolvida, guiadas por objetivos que se deslocavam entre diferentes limites disciplinares - da sociologia à psicologia, da organização à administração.
} 
As abordagens market-driven e market-driving de orientação para o mercado e inovação: proposição de um modelo integrado

\section{Considerações Finais}

A vantagem competitiva é um dos temas mais duradouros no âmbito da literatura da área de Administração Estratégica (HOOLEY et al. 2005). Dentre as diversas perspectivas conflitantes sobre os determinantes da vantagem competitiva, uma das que mais se destaca é a abordagem baseada em recursos da empresa (Resource Based View of the Firm), encabeçada por Barney (1991). Desenvolvida e sedimentada principalmente nos últimos 20 anos, essa abordagem postula que os recursos internos à organização comandam o desempenho da empresa e que a vantagem competitiva provém da posse e desenvolvimento de recursos que são, de alguma forma, superiores do que os dos competidores (BARNEY, 1991).

No desígnio do Marketing, a abordagem baseada nos recursos foi absorvida, despertando o interesse no papel dos recursos de marketing na criação de vantagens competitivas (BHARDWAJ; VARADARAJAN; FAHY, 1993). Contudo, o crescimento de trabalhos teóricos e conceituais sobre recursos de marketingnão se reflete, segundo Hooley et al. (2005), em investigações empíricas. Desta forma, muito da literatura normativa continua sem teste empírico e com observação limitada. Como exceção, destaca-se um recurso de marketing que tem recebido extensa atenção de estudos empíricos: a orientação para o mercado.

A despeito da proliferação de estudos na área, a literatura market-driving mostra ainda incontestes limitações. Primeiramente, há poucos trabalhos sobre o tema. Em segundo lugar, esses trabalhos constituem-se ou em descrições conceituais a partir de evidências do contexto empresarial (JAWORSKI; KOHLI; SAHAY, 2000; KUMAR; SCHEER; KOTLER, 2000), em estudos de caso isolados (HARRIS; CAI, 2002; TARNOVSKAYA; ELG; BURT, 2005), ou revisões de literatura enriquecidas por inputs de teóricos atuantes no marketing (HILLS; SARIN, 2003). Assim, há uma séria lacuna empírica a ser transposta no campo.

Em termos gerenciais, entende-se que o debate aqui delineado traga implicações importantes, considerando que a característica essencial do marketing repouse em sua relação direta e contínua com o ambiente externo, é de grande valia aprimorar o entendimento de como essa relação possa se dar. Assim, é de vital importância que gerentes e profissionais ligados ao marketing avaliem as possibilidades de atuar-se em relação ao ambiente externo de forma pró-ativa, questão para a qual o modelo apresentado - e suas consequentes administrações no campo empírico - poderá servir de substrato.

Nessa circunstância, primeiramente a varredura nos artigos relacionados ao tema evidenciou a ausência de um modelo prévio de pesquisa, de proposições relacionadas e de um instrumento de mensuração para o construto market-driving. Embora esforços como os de REAd - Edição 69 - Vol. 17 - N 2 - maio/agosto 2011 - p. 480-501 
Carrillat, Jaramillo e Locander (2004) - que oferecem uma estrutura conceitual pioneira para a estratégia market-driving - e Narver, Slater e MacLachlan (2004) - com a escala MOPRO (proactive market orientation) para mensuração da orientação market-driving - constituam-se em avanços importantes, não são suficientes para o desenvolvimento da área enquanto teoria.

O desenvolvimento aludido poderá ajudar a responder questões básicas como: (1) por que algumas organizações possuem mais habilidade em dirigir o mercado do que outras? (2) quais os efeitos de uma orientação market-driving sobre a performance? (3) teria uma orientação market-driving maior impacto sobre a performance do que a estratégia marketdriven? (4) sob que contingências ambientais a estratégia market-driving é mais eficiente? e (5) seria a orientação market-driven moderadora da relação market-driving sobre a performance? A articulação de respostas a essas e outras questões poderá representar a abertura de uma nova rota de pesquisa no âmbito da orientação para o mercado, direcionando análises sobre questões até aqui nada ou muito pouco exploradas.

O fortalecimento teórico do tema também poderá elucidar questões ainda nebulosas no âmbito da complementaridade driving/driven. (6) Será ela concomitante, como querem Day (1999), Jaworski, Kohli e Sahay (2000) e Carrillat, Jaramillo e Locander (2004)? (7) Ou sequencial, como defendem Harris e Cai (2003) e Kumar, Scheer e Kotler (2000)? Se o atual estágio de pesquisa não permite argumentar de forma definitiva na direção de um ou outro parecer, denota-se, portanto, uma questão em aberto, alvo potencial de futuras elucidações.

Outro ponto do ensaio teórico é o esforço de incluir modelos explicam os antecedentes das inovações, dado que para Freeman (2006) "um problema perene nas ciências sociais é a tendência de modelos teóricos e generalizações ganharem aceitação sem adequadas evidências ou testes empíricos, e de vez em quando, até sem qualquer evidência” (p.9).

Kirca, Jayachandran e Barden (2005), em seu meta análise com 61561 observações, encontraram empiricamente que uma das consequências mais fortes da orientação para o mercado é a inovação, especificamente o processo de inovação $(r=0,45)$ e a performance de novos produtos $(r=0,36)$. Assim, a necessidade de compreensão da orientação para o mercado e o processo de inovação e fundamental para o estabelecimento de uma vantagem competitiva.

A relação curvilinear proposta entre as orientações market-driven e customer-led necessita de maiores estudos. Análises futuras poderão utilizar o modelo para checar empiricamente se o ato de "ouvir os clientes em demasia" constitui-se, realmente, em um limitador estratégico marcante para as firmas. Isso ajudará a perceber o momento crítico da curva relacional hipotetizada na relação "foco no cliente" e "foco no mercado". 
As abordagens market-driven e market-driving de orientação para o mercado e inovação: proposição de um modelo integrado

Ainda como agenda futura, estimula-se o refinamento do modelo proposto por meio de pesquisas de campo, inicialmente de caráter exploratório e a posterior validação do construto por meio de etapa descritiva estruturada. Para tanto, faz-se necessária a construção de um instrumento de mensuração para o construto market-driving, ferramenta ainda inexistente e explicação para a lacuna empírica observada na área. Esse desenvolvimento é vital para a busca de respostas às sete indagações numeradas e outras que certamente surgirão.

A articulação das respostas descritas poderá representar a abertura de uma nova rota de pesquisa no âmbito da estratégia de orientação para o mercado, direcionando análises sobre questões até aqui muito pouco exploradas. Essa ampliação do arcabouço conceitual sobre a de orientação para o mercado trará à teoria valiosas contribuições teóricas, de importância inconteste para um campo de estudo ainda palco de inúmeros debates e controvérsias.

Indubitavelmente, portanto, entende-se que a concretização deste trabalho e o avanço dos objetivos ligados ao tema se constituirão em uma contribuição relevante para a produção de conhecimento sobre a orientação para o mercado, e, em maior perspectiva, para a disciplina de marketing como um todo.

\section{REFERÊNCIAS}

ARROW, K.J. The economics implications of learning by doing. Review of Economic Studies, 29, p. 155-173, 1962.

AVLONITIS, G. J.; GOUNARIS, S. P. Market orientation and company performance. Industrial Marketing Management, v. 26, n.5, p.385-402, 1997.

BARNEY, J. B. Firm Resources and Sustained Competitive Advantage. Journal of Management, v.17, n. 1, p. 99-120, 1991.

BHARDWAJ; Sundar G.; VARADARAJAN; P. Rajan; FAHY, John. Sustainable competitive advantage in service industries: a conceptual model and research propositions. Journal of Marketing, v. 57, October, p. 83-99, 1993.

BHUIAN, S. N. An empirical examination of market orientation in Saudi Arabian manufacturing companies. Journal of Business Research, v.43, n.1, p.13-25, 1998.

CANO, Cynthia R.; CARRILLAT, François A.; JARAMILLO, Fernando. A meta-analysis of the relationship between market orientation and business performance: evidence from five continents. International Journal of Research in Marketing, v.21, n.2, p.179-200, 2004. 
CARPENTER, Gregory S.; GLAZER, Rashi; NAKAMOTO, Kent. Readings on marketdriving strategies: towards a new theory of competitive advantage. Massachusetts: Addison-Wesley, 1997.

CARRILLAT, François A.; JARAMILLO, Fernando; LOCANDER, William. B. Marketdriving organizations: a framework. Academy of Marketing Science Review, v.5, p.1-14, 2004 .

CHRISTENSEN, Clayton M.; BOWER, Joseph L. Customer power, strategic investment, and the failure of leading firms. Strategic Management Journal, v.17, p. 197-218, Mar.1996.

CONNOR, Tom. Customer-led and market-oriented: a matter of balance. Strategic Management Journal, v. 20, p. 1157-1163, Dec. 1999.

DAY, George S. Misconceptions about market orientation. Journal of Market Focused Management, v.4, p.5-16, June, 1999.

DAY, George S. The Capabilities of market-driven organizations. Journal of Marketing, v.58, p.37-52, Out. 1994.

DAY, George S. The Capabilities of Market-Driven Organizations. Journal of Marketing, v. 58, n. 4, p 37-52, October, 1994.

DESHPANDÉ, Rohit; FARLEY, John U.; WEBSTER, Jr. Frederick E. Corporate culture, customer orientation, and innovativeness in japanese firms: a quadrad analysis. Journal of Marketing, v. 57, n.1, p. 23-37, Jan. 1993.

DOSI, Giovanni. Mudança Técnica e Transformação Industrial: uma teoria e uma aplicação à indústria dos semicondutores. Editora da Unicamp : Campinas, 2006.

FIGUEIREDO, A. Aprendizagem tecnológica e performance competitiva .Editora FGV; Rio de Janeiro, 2003.

FREEMAN, Christopher. Prefácio. In: Dosi, Giovanni. Mudança Técnica e Transformação Industrial: uma teoria e uma aplicação à indústria dos semicondutores. Editora da Unicamp : Campinas, 2006.

GAVA, R. . Desenvolvimento de um Modelo e de Proposições de Pesquisa para a Formulação e Implementação da Estratégia Market-Driving de Orientação Para o Mercado: Um Estudo Exploratório em Diferentes Indústrias. In: Enanpad 2007, 2007, Rio de Janeiro. Anais do Enanpad, 2007. 
As abordagens market-driven e market-driving de orientação para o mercado e inovação: proposição de um modelo integrado

GAVA, R. ; SILVEIRA, T. . A Orientação para o Mercado sob a Ótica da Empresa e dos Clientes na Indústria Hoteleira Gaúcha. RAC. Revista de Administração Contemporânea, v. 11, p. 49-69, 2007.

HAMEL, Gary; PRAHALAD, C. K. Corporate Imagination and Expeditionary Marketing. Harvard Business Review, p. 81-92, Jul./Aug. 1991.

HAN, Jin K.; KIM, Namwoon; SRIVASTAVA, Rajendra K. Market orientation and organizational performance: is innovation a missing link?. Journal of Marketing, v. 62, n. 4, p. 30-45, Oct. 1998.

HARRIS, Lloyd. C.; CAI, Kai Y. Exploring market driving: a case study of De Beers in China. Journal of Market - Focused Management, v. 5, n. 3, p. 171-196, Set. 2002.

HOMBURG, C.; PFLESSER, C.A. Multiple-layer model of market-oriented organizational culture: measurement issues and performance outcomes. Journal of Marketing Research, v.27, p.449-462, November, 2000.

HOOLEY, Graham J.; GREENLEY, Gordon E., CADOGAN, John W.; FAHY, John. The performance impact of marketing resources. Journal of Business Research , v.58, n. 1; p. 18-36, 2005.

HURLEY, Robert F.; HULT, G. Thomas M. Innovation, market orientation, and organizational learning: an integration and empirical examination. Journal of Marketing, v. 62, n. 3, p. 42-54, July 1998.

IPIRANGA, A.R. Os Arranjos e Sistemas Produtivos Territoriais entre Aprendizagem, Inovação e Cultura. Anais do EnAnpad, 2006, Salvador - BA.

JAWORSKI, Bernard J.; KOHLI, Ajay K.; SAHAY, Arvind. Market-driven versus driving markets. Journal of the Academy of Marketing Science, v. 28, p. 45-54, Jul. 2000.

JOHNSON, Jean L.; LEE, Ruby P.; SAINI, Amit; GROHMANN, Bianca. Market-focused strategic flexibility: conceptual advances and an integrative model. Journal of the Academy of Marketing Science, v. 31, n. 1, p. 74-89, Winter 2003.

KIRCA, A. H.; JAYACHANDRAN S.; BEARDEN, W. O. Market Orientation: A MetaAnalytic Review and Assessment of Its Antecedents and Impact on Performance. Journal of Marketing, v. 69, n.2, p.24-41, 2005.

KIRCA, Ahmet H.; JAYACHANDRAN, Satish; BEARDEN, William. O. Market orientation: a meta-analytic review and assessment of its antecedents and impact on performance. Journal of Marketing, v. 69, p. 24-41, Apr. 2005. 
KOHLI, Ajay K.; JAWORSKI, Bernard J. Market orientation: the construct, research propositions, and managerial implications. Journal of Marketing, vol. 54, p. 1-18, Abr. 1990.

KUMAR, Nirmalya, SCHEER, Lisa; KOTLER, Philip. From market-driven to marketdriving. European Management Journal, v. 18, nº 2, p. 129-141, 2000.

KUMAR, Nirmalya. The revolution in retailing: from market driven to market driving Long Range Planing, v. 30, p. 830-836, 1997.

LANGERAK, Fred. What is the predictive power of market orientation? ERIM Report Series - Research in Management, ERS-88-MKT, 2002.

MAVONDO, Felix T.; CHIMHANZI, Jacqueline; STEWART, Jillian. Learning orientation na marketing orientation: relationship with innovation, human resource practices and performance. European Journal of Marketing, v. 39, n. 11/12, p. 1235-1263, 2005.

MENNA, Hélio L. Orientação para mercado e performance: evidências em empresas gaúchas de varejo de confecções masculinas. In: XXV Encontro Anual da Associação Nacional dos Programas de Pós-graduação em Administração - ENANPAD, 2001. Anais. Marketing. Campinas SP: Associação Nacional de Programas de Pós-Graduação em Administração ANPAD, 2001. (Texto integral em CD-ROM dos Anais do $25^{\circ}$ ENANPAD).

NARVER, John C.; SLATER, Stanley F. The effect of a market orientation on business profitability. Journal of Marketing, v. 54, p. 20-35, Out. 1990.

NARVER, John C.; SLATER, Stanley; MacLACHLAN, Douglas. Responsive and proactive market orientation and new-product success. The Journal of Product Innovation Management, v. 21, p. 334-347, 2004.

NELSON, R.; WINTER, S. Uma teoria evolucionária da mudança tecnológica. Editora Unicamp, Campinas 2005.

PELHAM, A. M. Mediating influences on the relationship between market orientation and profitability in small industrial firms. Journal of Marketing Theory and Pratice, v. 5, n. 3, p. 55-76, 1997.

PITT, L.; CARUANA, A.; BERTHON, P. R. Market orientation and business performance: some European Evidence. International Marketing Review, v. 13, n. 1, p. 5-18, 1996. 
As abordagens market-driven e market-driving de orientação para o mercado e inovação: proposição de um modelo integrado

RÉVILLION, Anya S.P. Inter-relações entre orientação para o cliente, cultura organizacional e cultura nacional: proposta de um modelo. Revista de Ciências da Administração, v.5, n.10, p.75-92, 2003.

ROZEBOOM, W.W. Mediation variables in scientific theory. Psychological Review, v.63, n.4, p.249-264, 1956.

RUEKERT, Robert W. Developing a market orientation: an organizational strategy perspective. International Journal of Research in Marketing, v.9, p. 225-245, 1992.

SHAPIRO, Benson P. What the hell is 'market-oriented'? Harvard Business Review, 66: 119-25, Nov./Dec. 1988.

SLATER, S.F.; NARVER, J.C. Does competitive environment moderate the market orientation-performance relationship? Journal of Marketing, v. 58, n.1, p. 46-55, January. 1994.

SLATER, Stanley F.; NARVER, John C. Market Orientation and the Learning Organization. Journal of Marketing, v. 59, n. 3, p. 63-74, 1995.

SLATER, Stanley F; NARVER, John C., Customer-led and market-oriented: let's not confuse the two. Strategic Management Journal, v. 19, p. 1001-1006, 1998.

SLATER, Stanley, NARVER, John C. Market-oriented is more than being customer-led. Strategic Management Journal, v. 20, p. 1165-1168, 1999.

SUBRAMANIAN, R.; GOPALAKRISHNA, P. The market orientation-performance relationship in the context of a developing economy: an empirical analysis. Journal of Business Research, v. 53, n. 1, p. 1-13, 2001.

TARNOVSKAYA, Veronika; ELG, Ulf; BURT, Steve. The role of corporate branding in a market driving strategy. Workin Paper Series - Lund Institute of Economic Research, 2005/02.

VARADARAJAN, Rajan P.; CLARCK, Terry; PRIDE, William M. Controlling the uncontrollable: managing your market environment. Sloan Management Review, v. 33, n. 2, p. 39-47, 1992.

WEBSTER, Jr. Frederick E. Defining the new marketing concept. Marketing Management, v. 2, n. 4, p. 22, 1994. 
WRIGHT, N.D.; PEARCE, J.W.; BUSBIN, J.W. Linking customer service orientation to competitive performance: does the marketing concept really work? Journal of Marketing Theory and Practice, p.23-34, Fall 1997.

ZEITHAML, Carl P.; ZEITHAML, Valarie A. Environmental management: revising the marketing perspective. Journal of Marketing, v. 48, p. 46-53, Spring 1984.

\title{
RESUMO
}

Direcionamentos recentes da teoria de orientação para o mercado têm apontado para a natureza eminentemente reativa das estratégias relacionadas. Argumenta-se que a abordagem tradicional - market-driven - seja excessivamente determinista e de forte conotação adaptativa perante o ambiente externo. Essa visão passa a ser complementada por uma nova perspectiva - market-driving -, pressupondo a possibilidade de que as estruturas e o comportamento do mercado possam ser modelados pelas organizações. Entretanto, ainda são incipientes os esforços na construção de um modelo de referência que possa nortear futuras pesquisas no âmbito dessa estratégia. É nessa problemática que se insere o objetivo do presente trabalho, em vistas de apresentar um modelo teórico e proposições de pesquisa sobre o tema, baseados em intensiva revisão de literatura. Como conclusão, o modelo construído permite vislumbrar os antecedentes da estratégia market-driving, suas respectivas ações de implementação e variáveis correlatas. Também se verifica a natureza complementar dessa abordagem em relação à postura tradicional de orientação. Ao final, apresentam-se as implicações acadêmicas e gerenciais do presente estudo, bem como a agenda para pesquisas futuras.

Palavras-Chave: Estratégia; Market-Driving; Orientação para o Mercado

\section{MARKET-DRIVING STRATEGY FOR MARKET ORIENTATION: A THEORETICAL MODEL AND SUGGESTIONS FOR RESEARCH}

\begin{abstract}
Recent developments in the market orientation theory have been indicating for the reactive nature of the related strategies. It argues that the traditional approach, market-driven, is excessively determinism and of strong adaptative connotation in front of external atmosphere. That vision becomes complemented by a new perspective, market-driving, presupposing that the market structures and its behavior can be modeled by the organizations. However, this theory is still incipient in the construction of a reference model that can orientate future research using that strategy. This paper looks for fulfill this gap by presenting a theoretical model and some research propositions on the theme, based on intensive literature review. As conclusion, the model allows to analyze the antecedents of the market-driving strategy, review some actions to be implemented and verify correlated variables. The complementally nature of that approach is also verified in relation to the traditional posture of orientation. At the end, some academic and managerial implications as discussed, as well as, the agenda for future research.
\end{abstract}

Keywords: strategy; market-driving; market orientation 